This is the accepted manuscript, which has been accepted by IEEE for publication @ 2014. Personal use of this material is permitted. Permission from IEEE must be obtained for all other uses, in any current or future media, including reprinting/republishing this material for advertising or promotional purposes, creating new collective works, for resale or redistribution to servers or lists, or reuse of any copyrighted component of this work in other works. The full reference is:

"Tuning of a Damping Controller for Multi-terminal VSC-HVDC Grids using the Probabilistic Collocation Method"

R. Preece, J.V. Milanović

IEEE Transactions on Power Delivery Special Issue on 'HVDC Systems and Technologies', vol. 29, issue 1, pp. 318-326, 2014

Digital Object Identifier: 10.1109/TPWRD.2013.2258945 


\title{
Tuning of a Damping Controller for Multi- terminal VSC-HVDC Grids using the Probabilistic Collocation Method
}

\author{
R. Preece, Graduate Student Member, IEEE, and J. V. Milanović, Fellow, IEEE
}

\begin{abstract}
This paper presents a robust probabilistic controller tuning method to improve the damping of critical system modes through the modulation of active power injected by a voltage source converter based multi-terminal high voltage direct current (VSC-MTDC) grid. This methodology first establishes the probabilistic locations of the critical modes based on the known variation in power system operating conditions. Following this, the modal linear quadratic Gaussian (MLQG) controller structure is tuned for a set of probabilistic values of critical eigenvalues. The controller's performance following small disturbances in the network for wide ranging operating conditions is compared with the conventionally tuned MLQG controller designed for the nominal system operating point. The Probabilistic Collocation Method is shown to facilitate robust probabilistic tuning without the need for large numbers of full system linearizations. The test system used incorporates a large wind farm with variable power output connected to the meshed AC network through the VSC-MTDC grid.
\end{abstract}

Index Terms - electromechanical modes, modal LQG control, multi-terminal VSC-HVDC, offshore wind, robustness, power oscillation damping, probabilistic collocation method.

\section{INTRODUCTION}

$\mathrm{F}$ UTURE power transmission networks will operate closer to stability limits with points of power injection subject to rapid change as renewable sources of generation proliferate. With increasing power demands and already strained AC transmission lines, high voltage direct current (HVDC) links are likely to see ever increasing penetration. Voltage Source Converter (VSC) based HVDC systems are becoming increasingly competitive (both financially and in terms of power losses) and provide many benefits over classic Line Commutated Converter (LCC) based HVDC systems. One such benefit is the ready extension to multi-terminal (MTDC) configuration.

A VSC-MTDC grid would allow DC interconnections between multiple connection points in any number of asynchronous AC power networks. A meshed VSC-MTDC system such as this would allow easy connection of distant offshore wind farms with existing AC systems whilst also

This work was support in part by the Engineering and Physical Sciences Research Council (EPSRC) and in part by National Grid Plc.

R. Preece and J.V. Milanović are with the School of Electrical and Electronic Engineering, The University of Manchester, PO Box 88, Manchester, M60 1QD, UK. (email: r.x.preece@gmail.com, milanovic@manchester.ac.uk). providing a means of increased energy trading between the interconnected system operators. There is also increasing discussion about a pan-European MTDC 'supergrid' which would allow vast interconnection between the various areas of the European AC system [1]. Such a system would allow not only improved energy trading and integration of renewable energy sources, but also the potential for enhancement of system stability.

Although no VSC-MTDC systems currently exist, there are great expectations surrounding their future development [2], and there has been some research interest in the field. This includes work on protection requirements [3], DC voltage control strategies [4], and the stability concerns surrounding the integration of MTDC grids [5]. Despite this interest in VSC-MTDC grids, there is little work investigating the enhancement of system stability using the fast controllable converter technology. Modulation of the active power injection into the AC grid can be utilized to damp troublesome persistent post-disturbance power system oscillations.

Previous investigations have demonstrated the effectiveness of using HVDC active power modulation for power oscillation damping (POD) purposes [6, 7], though only with point to point HVDC links. The controller utilized within this study is wide area measurement system (WAMS) based and takes signals from across the entire AC system to effectively stabilize the network. The controller takes a Modal Linear Quadratic Gaussian (MLQG) structure which has been demonstrated to be effective for POD through HVDC systems $[6,7]$. This design allows targeted action of specified system modes (in this study, low frequency electromechanical oscillations) whilst leaving the remainder unaffected, making it particularly suitable as a supplementary system controller. It should be noted that the robust tuning methodology presented could be applied to any controller design which uses a linearized power system model (e.g., $H_{\infty}$ designs) and is not dependent upon the MLQG controller used within this study.

Through the use of probabilistic studies, the most likely open loop modal positions can be determined. Formation of the controller design process using modal canonical forms of the power system state space model allows manipulation of the power system representation so these probabilistic modes can be used during the design process.

Furthermore, the use of the Probabilistic Collocation Method (PCM) allows efficient identification of the 
probabilistic values of the critical system eigenvalues without the need for vast quantities of full system linearizations. As such, the probabilistic tuning process can be readily completed and improved controller robustness is achieved.

The work presented in this paper establishes that the use of a probabilistic system representation during WAMS-based POD controller design will result in final controllers which are more robust to operational uncertainties. The suitability of the PCM to efficiently produce this probabilistic representation in large power systems is also presented for the first time. The proposed methodologies are not limited by controller type and structure, nor by the uncertainties considered, and as such they are widely applicable to power system stability studies.

\section{Probabilistic CONTROLler TUNING METHODOLOGY}

The locations of electromechanical modes vary as the power system operating point changes. Although a controller may be designed for the nominal operating point of a network, real operating conditions will be defined by factors including load variation, renewable generation availability and energy market fluctuations. It therefore makes more sense to use probabilistic mode locations (rather than the mode locations at the nominal operating point) when designing a damping controller. This will ensure that the controller action is targeted at the most likely frequencies associated with the modes to be damped.

\section{A. Determining Probabilistic Mode Locations}

Due to the nonlinearities of power systems, the most probable modal locations do not necessarily correspond to the most probable operating conditions. The probability distributions for the open loop locations of the electromechanical modes must be determined using the Monte Carlo approach and many system linearizations. Alternatively, more efficient computational techniques can be used, such as Latin hypercube or low discrepancy sequence based sampling, or the Probabilistic Collocation Method as will be later demonstrated within this paper.

Any distribution can be utilized to generate the operating conditions for a power network (including conditional distributions or historical information). In this study, generator power output and load demand are assumed to be normally distributed, wind speed follows a Weibull distribution, and VSC-MTDC converter station power injection follows a uniform distribution. Operating conditions are selected by drawing operating points from the specified distributions. Only feasible operating conditions (with converged load flows and stable open loop modes) are considered. At this stage, optimal power flow (OPF) was not considered, however the concept presented is still valid and could be implemented with OPF if desired.

Once the probability distributions for each electromechanical mode have been established, probabilistic modes can be defined as the most probable values for the electromechanical modes of interest. The peak values from the probability distributions for both the real and imaginary parts of the modes are selected. The use of these probabilistic modes during the controller design, rather than the nominal modes, leads to a controller which is more robust to the changing operating conditions of the power system.

\section{B. Probabilistic System Representation}

The linearized state space power system model described by (1) and (2) can be rewritten in the modal canonical form of (3) and (4).

$$
\begin{gathered}
\dot{\mathbf{x}}=\mathbf{A x}+\mathbf{B u} \\
\mathbf{y}=\mathbf{C x} \\
\dot{\mathbf{z}}=\mathbf{A}_{M} \mathbf{z}+\mathbf{B}_{M} \mathbf{u} \\
\mathbf{y}=\mathbf{C}_{M} \mathbf{z}
\end{gathered}
$$

Where the modal variables $z$ directly correspond to the system modes $\lambda_{i}$ (where $i=1 \ldots n$ and the power system is represented by an $n^{\text {th }}$ order model). The modal variables $z$ are mapped to the standard state variables $\boldsymbol{x}$ as $\mathbf{z}=\mathbf{M x}$ where $\mathbf{M}$ is the modal transform matrix. $\mathbf{M}$ is obtained using Real Schur Decomposition and relates to the matrix of right eigenvectors $\Phi$ as $\mathbf{M}=\Phi^{-1}$. The modal state matrices are defined as $\mathbf{A}_{M}=\mathbf{M A M} \mathbf{M}^{-1}, \mathbf{B}_{M}=\mathbf{M B}$, and $\mathbf{C}_{M}=\mathbf{C M}^{-1}$.

The modal transition matrix $\mathbf{A}_{M}$ is diagonal with the structure (5).

$$
\mathbf{A}_{M}=\operatorname{diag}\left\{\left[\lambda_{1} \cdots \lambda_{n_{r}}\right],\left[\begin{array}{cc}
\sigma_{1} & \omega_{1} \\
-\omega_{1} & \sigma_{1}
\end{array}\right] \cdots\left[\begin{array}{cc}
\sigma_{n_{c}} & \omega_{n_{c}} \\
-\omega_{n_{c}} & \sigma_{n_{c}}
\end{array}\right]\right\}
$$

where $\lambda_{1} \cdots \lambda_{n_{r}}$ are the purely real eigenvalues; $n_{r}$ is the number of real eigenvalues; the $2 \times 2$ block diagonals $\left[\begin{array}{cc}\sigma_{1} & \omega_{1} \\ -\omega_{1} & \sigma_{1}\end{array}\right] \ldots\left[\begin{array}{cc}\sigma_{n_{c}} & \omega_{n_{c}} \\ -\omega_{n_{c}} & \sigma_{n_{c}}\end{array}\right]$ correspond to the complex pairs of eigenvalues $\lambda_{i}=\sigma_{i} \pm j_{i}$; and $n_{c}$ is the number of complex eigenvalue pairs.

With the system represented in a diagonal form, the mode locations for the nominal system can be modified to represent the probabilistic modes which have already been determined. Values in $\mathbf{A}_{M}$ are altered for the critical electromechanical modes of interest (to create $\mathbf{A}_{M}^{\text {prob }}$ ) and the control design can be completed on a probabilistic system representation.

\section{The Probabilistic COLlocation MethoD}

The probabilistic collocation method (PCM) can be used to approximate the statistical distribution of a system output based upon a set of known uncertainties. This is completed by using a much smaller number of system simulations than is required for traditional Monte Carlo (MC) approaches. Other methods for efficient sampling of the search space include the use of low discrepancy sequences, $\Lambda \Pi_{\tau}$ sequences and Latin hypercube techniques [8-10]. However these techniques evenly sample the whole search space, whereas the PCM specifically focuses upon those regions most likely to occur. Methods such as two point estimates can also provide accurate results with very few full samples [11], however they are 
constrained by their presumption that the output of interest linearizations and collocation points with the greatest

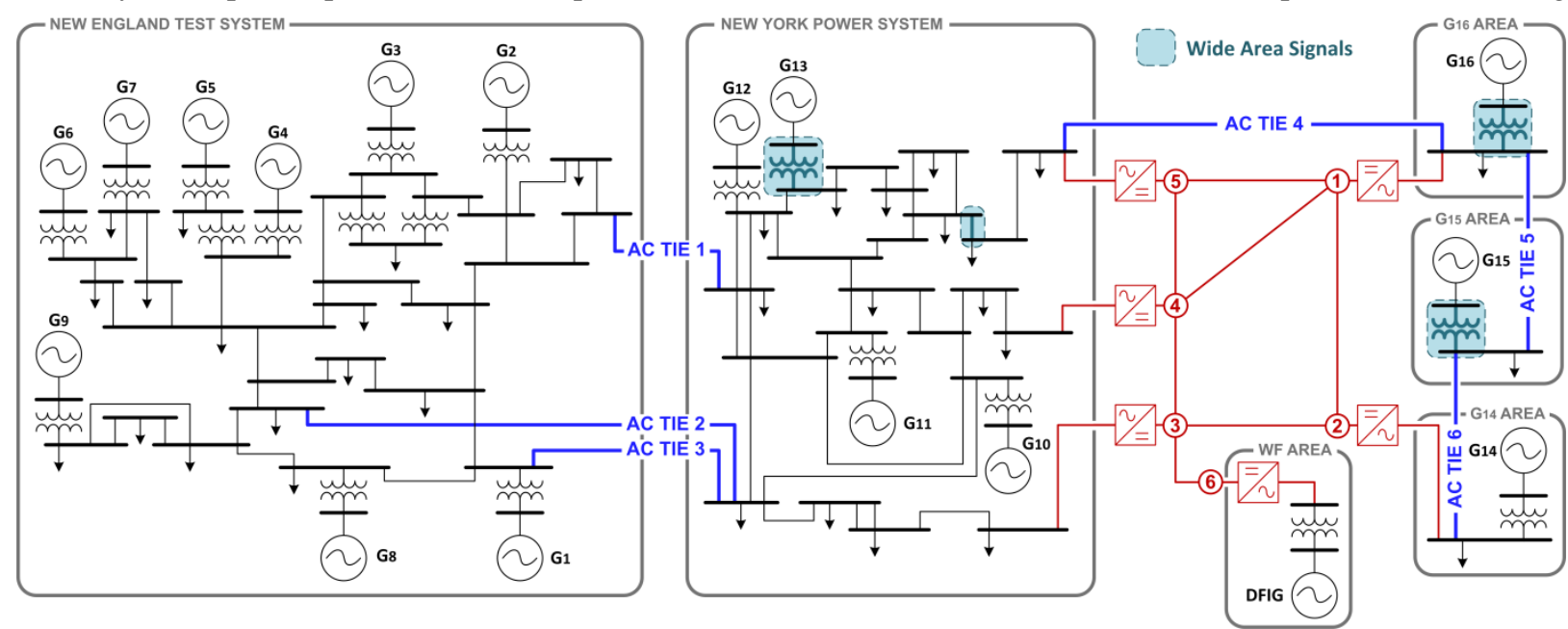

Fig. 1. Modified NETS NYPS test network, including multi-terminal VSC-HVDC grid and large offshore wind farm.

will follow a predetermined distribution (typically Normal). This is not necessarily the case with practical non-linear power systems, and is not a constraint on the PCM approach.

More fully explained in [12], the approximated response $\hat{Y}$ is modeled as a polynomial function of the uncertain parameter set $\Psi$, as shown in (6).

$$
\hat{Y}=g(\Psi)
$$

The PCM function can be used as a computationally inexpensive substitute for running a full linearization of the power system during standard MC based uncertainty studies. Central to the PCM is the selection of the representative polynomial function, and the specification of the points at which full linearization is required (to gather the data and build the model).

\section{A. PCM polynomial model function}

The model function $g(\Psi)$ is formed as a sum of products of orthogonal polynomials which represent the known probability distributions of the uncertain system parameters, weighted by a set of coefficients $\boldsymbol{K}$. These orthogonal polynomials can take a standard form (such as the Hermite polynomials used to represent normally distributed parameters) or can be derived for any distribution using recursive methods [13].

\section{B. Full linearization operating point selection}

PCM model functions can be of varying order, dependent upon the order of the polynomials that are used in the sum of products. As higher order models, or a greater number of uncertainties, are considered the possible number of combinations of orthogonal polynomials increases. This requires the calculation of an increased number of coefficients.

Full system linearizations are run at specified operating points to provide the data from which the set of coefficients $\boldsymbol{K}$ can be deduced. These collocation points are selected by taking inspiration from the Gaussian quadrature technique of estimating integrals [13]. The roots of higher order polynomials are chosen as the points at which to perform full probability of occurring are selected. The accuracy of the PCM model is concentrated in the regions defined by the $p d f s$ as most likely to occur. For large systems this requires calculating all possible permutations of operating conditions and ordering by their probability of occurrence. These are then iterated through (performing load flow to ensure convergence) in order to determine which operating conditions are selected; such that the matrix inversion required to calculate $\boldsymbol{K}$ is possible.

\section{TEST SYSTEM}

The proposed methodology for improving damping controller robustness has been tested on a modified version of the New England Test System and New York Power System (NETS NYPS), shown in Fig. 1. This test network is commonly used for studies into power oscillations and displays multiple inter-area modes. System analysis and simulations are all performed within the MATLAB/Simulink environment making use of modified MATPOWER [14] functions to perform initial load flows.

\section{A. AC System Details}

Two AC inter-area ties have been removed and a meshed VSC-MTDC grid has been installed between the NYPS, G14 and G16 areas. Generators G1-8 are under slow DC excitation (IEEE-DC1A) only, whilst G9 is equipped with a fast acting static exciter (IEEE-ST1A) and a Power System Stabilizer (PSS). The remaining generators (G10-16) are under constant manual excitation. All generators are represented by full sixth order models. Loads are modeled as constant impedance. Full system details, generator and exciter parameters are given in [15] with PSS settings for G9 taken from [16].

\section{B. VSC-MTDC System Details}

The included six-converter VSC-MTDC network represents a possible interconnection between multiple areas of an AC network. Locations for MTDC nodes 1-5 have been selected as the same buses which previously fed AC inter-area ties. Each converter station is modeled as an injection of active and reactive power [17]. When interested in electromechanical 
oscillations with typical frequencies of $0.2-2 \mathrm{~Hz}$, the very fast dynamics associated with the semiconductor device switching operations can be neglected [17]. Converter station controllers are included as described in [7] and DC lines are modeled as presented in [18].

There is some work surrounding the use of voltage-droop characteristics with MTDC grids, for example [4], demonstrating its importance during transient events or outage scenarios. As the studies performed here are concerned with post-disturbance modulation of active power flow in order to damp AC system oscillations, the simpler use of a slack DC bus is used (the slack converter VSC-1 regulating voltage).

Note that further work is currently being carried out into the application of MTDC-based POD control incorporating the voltage-droop characteristics that would be seen in practical installations. Possible implementations include the direct overlay of the POD scheme with the droop characteristic, and a switch in control mode following the identification of AC oscillations in need of supplementary damping.

Nominal values of active power injection for VSC-HVDC converters 2-5 are set based upon previous $\mathrm{AC}$ tie line power flows. Active power flow injection at VSC-6 is determined by the output of the connected wind farm. Reactive power injection at the MTDC nodes 1-5 is regulated at zero. At VSC6 , reactive power is supplied by the converter as required to support the wind farm. At each MTDC node regulating power flow excluding the wind farm (i.e. VSC converters 2-5) there exists a supplementary input signal $\Delta P^{\text {ref }}$ which can be used to modulate the active power reference set points for stabilizing purposes. Modulation is limited to $10 \%$ of rated capacity, with all MTDC line parameters and converter controller settings are provided in the Appendix.

\section{Wind Farm System Details}

In addition to the VSC-MTDC grid, a further modification has been made to the network with the inclusion of a large wind farm. This $300 \mathrm{MW}$ farm is connected to the AC network through the VSC-MTDC grid (as is shown in Fig. 1).

For the purpose of the studies performed, it has been assumed that power output from the wind farm is constant at for each investigated operating point. VSC-6, to which the wind farm in connected, operates with frequency-voltage control. It assumed that the converter is able to maintain a constant $\mathrm{AC}$ voltage such that all power produced by the wind farm is transferred to the VSC-MTDC system. This assumption holds provided the MTDC system voltage does not deviate considerably. With the focus on the post disturbance stabilization of the main AC system and controller robustness to variable operating conditions (and not the fast transient performance of the VSC-MTDC grid), this is an acceptable simplification.

\section{POD Controller Design}

Small signal analysis of the full test system as described (at the nominal operating point) reveals four electromechanical modes with low frequency and poor damping factors $(\zeta<5 \%)[16]$, detailed in Table 1 . All other electro- mechanical modes are adequately damped and require no further control action. The $\Delta P^{\text {ref }}$ inputs to the VSC-MTDC grid are to be used to attempt to improve the system stability via a supplementary POD controller.

The probabilistic controller tuning approach proposed here can be used with any WAMS controller design based upon a linearized model representation of the power system. Within this study, a WAMS based controller is designed using the Modal Linear Quadratic Gaussian structure (detailed in [7, 19]). This controller structure allows targeted action on specified modes, leaving those which are already adequately damped unaffected. All four low frequency modes are targeted. Following a modal observability study of the entire network, optimal controller inputs are selected as active power flows through the lines highlighted in Fig. 1. Controller outputs are the $\Delta P^{\text {ref }}$ signals to MTDC nodes 2-5.

TABLE 1

LOW FREQUENCY ELECTROMECHANICAL MODE DETAILS FOR TEST SYSTEM

\begin{tabular}{lccc}
\hline & $\begin{array}{c}\text { Eigenvalue (pu) } \\
\lambda=\sigma \pm j \omega\end{array}$ & $\begin{array}{c}\text { Damping Factor (\%) } \\
\zeta=-\sigma / \sqrt{\sigma^{2}+\omega^{2}}\end{array}$ & $\begin{array}{c}\text { Frequency (Hz) } \\
\omega / 2 \pi\end{array}$ \\
\hline \hline Mode 1 & $-0.071 \pm j 1.588$ & 4.47 & 0.253 \\
Mode 2 & $-0.127 \pm j 2.744$ & 4.61 & 0.427 \\
Mode 3 & $-0.167 \pm j 3.917$ & 4.27 & 0.623 \\
Mode 4 & $-0.237 \pm j 4.900$ & 4.81 & 0.780 \\
\hline
\end{tabular}

Signal delays of $375 \mathrm{~ms}$ are assumed for system measurements (modeled as $2^{\text {nd }}$ order Padé approximations during system linearization). Controller output signals are assumed to have negligibly small delays (i.e. hard-wired fiber connections laid to all MTDC converter stations). It is sometimes necessary to reduce the order of the plant model when using LQG controller designs to avoid ill conditioning when solving high order matrix Riccati equations. As this problem was not experienced, controller design was carried out using the full linearized system model.

The nominal controller was designed using fine tuning of weighting functions to first achieve equal target damping factors of $20 \%$ to Modes 1-4. Weights were then reduced uniformly to limit the gain of the largest singular value of the transfer function of the Linear Quadratic Regulator (LQR) to $6 \mathrm{~dB}$ to improve robust stability properties [20]. The final controller order was then reduced to lower the online computational burden (from $235^{\text {th }}$ to $32^{\text {nd }}$ order). The bound on final controller order was set to limit low frequency mode damping factor degradation to no more than 5\%. Final nominal closed loop damping factors for Modes 1-4 were 12.3\%, 13.1\%, $11.3 \%$ and $11.8 \%$ respectively.

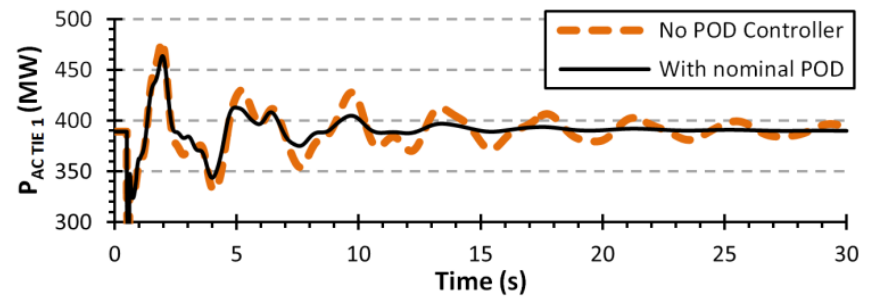

Fig. 2. Example transient performance of nominal controller at the nominal operating point following a three-phase self-clearing $100 \mathrm{~ms}$ fault within the NYPS area of the test network. 
As can be seen from the transient plot of Fig. 2, these are sufficient to stabilize the network oscillations quickly following disturbances. Similar results could be achieved using alternative controller designs.

\section{APPliCATION OF PROBABILISTIC CONTROLlER TUNING}

The details relating to the application of the probabilistic tuning methodology on the outlined test system are presented.

\section{A. Variation in System Operating Conditions}

Generators are modeled as having non-varying voltage and normally distributed active power output. System loads are modeled with constant power factor and normally distributed active power demand. The given nominal set point is assumed to represent $80 \%$ of maximum loading, with full loading signifying a $+3 \sigma_{\psi}$ increase from the mean values $\mu_{\psi}$.

VSC-MTDC power injection values are set as uniformly distributed around the nominal values. Nodes 2-5 have nominal values of power injection into the AC grid of -600 , 400, 350, and $500 \mathrm{MW}$ respectively. The uniform distribution is limited to $\pm 100 \mathrm{MW}$ at each node.

The generation capacity of the wind farm is determined by the wind speed $v$. For these studies, wind speed is a random variable following a Weibull distribution, as described by (7).

$$
f(v)= \begin{cases}\frac{k}{\varphi}\left(\frac{v}{\varphi}\right)^{k-1} e^{-(v / \varphi)^{k}} & v \geq 0, \\ 0 & v<0 .\end{cases}
$$

Where $k$ is the shape parameter and $\varphi$ is the scale parameter (commonly signified by $\lambda$ but called $\varphi$ here to avoid confusion). In this study, values for these parameters were sourced from [21] with $k=2.2$ and $\varphi=11.1$, implying a mean wind speed of $\bar{v}=9.83 \mathrm{~ms}^{-1}$. The wind farm consists of 150 Vestas V-80 2 MW turbines [22]. The total power produced is calculated by randomly selecting a wind speed, feeding this value into the power curve for the V-80 turbine, and then finally scaling the individual turbine output to the capacity of the whole wind farm, neglecting wake effects.

Probabilistic studies are performed using 5000 system linearizations and a standard MC approach, drawing operating parameters from the distributions outlined.

\section{B. Identification of Electromechanical Modes}

The robustness of the designed supplementary POD controllers was assessed through small disturbance analysis. This required the use of classification tools to ensure accurate identification of the critical system modes. A Naïve Bayes classifier [23] was used to identify the necessary modes based upon their damping, frequency and system state modal participation factors.

\section{REsults of Probabilistic CONTROLLER TUNING}

\section{A. Performance of Nominal Controller}

The nominal controller (designed as described in Section IV.D for the power system operating at the nominal operating point) was tested to assess the closed loop robustness to varying operating conditions. Fig. 3 shows the closed loop locations of the four low frequency modes with the nominal controller installed.

Modes 1 and 4 maintain damping factors greater than 5\% for all operating conditions. This is also true for the vast majority of operating conditions for Mode 3, which sees the damping factor drop below $5 \%$ in just 30 of the 5000 simulations; i.e. $P(\zeta<5 \%)=0.6 \%$. Mode 2 , however, does not have such satisfactory performance with $P(\zeta<5 \%)=$ $27.1 \%$. Although modes 1,3 , and 4 may be considered suitably damped, Mode 2 is not, and the probabilistic tuning method may improve the controller performance.

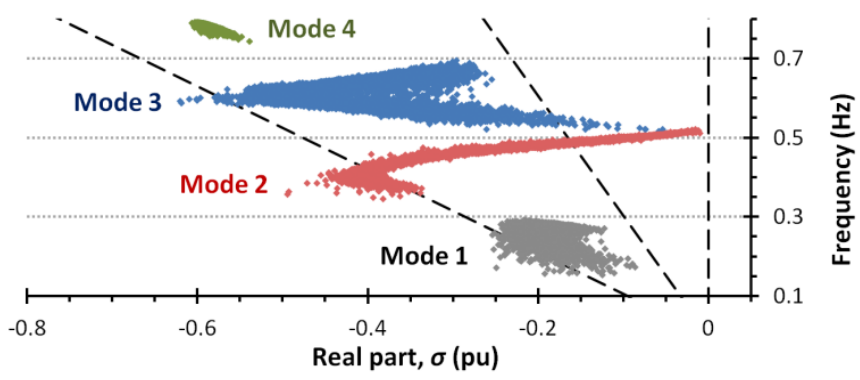

Fig. 3. Closed loop low frequency mode locations with nominal controller. Dashed lines show damping factors of $0 \%, 5 \%$ and $15 \%$.

\section{B. Nominal Open Loop Modes}

By assessing the position of the nominal open loop low frequency modes, we can begin to judge whether the use of probabilistic tuning will have an impact on the controller's robustness. Fig. 4 displays the spread of open loop mode positions seen due to the variation in operating conditions.

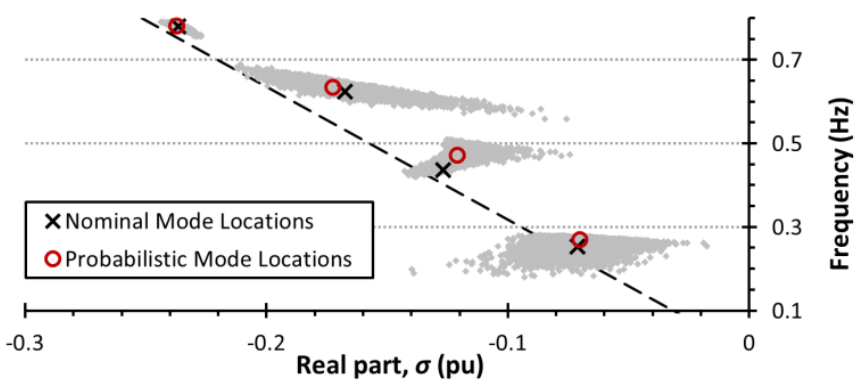

Fig. 4. Open loop low frequency mode locations, including the nominal and probabilistic values.

Also shown in Fig. 4 are the nominal open loop mode positions and the probabilistic mode locations based on the modal spread observed. It can be seen that the nominal mode locations are not always representative of the probabilistic locations. It is clear that the greatest disparity between the two is for Mode 2 (which displays the poorest closed loop performance). Use of the probabilistic values should improve the controller's robust performance with respect to the damping of Mode 2. This is performed in the sequel to demonstrate the benefits of this probabilistic system representation.

This critical mode spread is dependent on the variation in operating conditions, and variation between the probabilistic and nominal mode locations could be much greater than shown in Fig. 4. This disparity is also clearly dependent on the designation of the nominal operating point. 


\section{Performance of the Probabilistic Controller}

The process of designing the controller was completed once more using the probabilistic values for Mode 2: $\lambda_{\text {prob }}^{\text {Mode }}=$ $-0.121 \pm j 2.96$ (obtained as described in Section II.A). Again, the target damping was set at $20 \%$ per mode, the LQR singular value gain limited to $6 \mathrm{~dB}$, and the final controller reduced to $32^{\text {nd }}$ order. This probabilistic controller was then reassessed against the full range of defined operating conditions.

Fig. 5 presents a box and whisker plot of the damping factors with the nominal controller and the probabilistic controller installed. The boxes represent the $25^{\text {th }}$ and $75^{\text {th }}$ percentiles; the bar within the box shows the median; the whiskers show the minimum and maximum values; and the notches on the whiskers display the $2.5^{\text {th }}$ and $97.5^{\text {th }}$ percentiles (i.e. $95 \%$ of the data is contained within these notches).

It can be seen that through using the probabilistic mode locations, the robustness of the controller has been improved. The damping factor for Mode 3 is largely unchanged, and that of Mode 4 is slightly reduced but still always greater than $10 \%$. Mode 1 also experiences slightly reduced damping but $P(\zeta<5 \%)$ is still equal to zero. These slight changes are due to variations in the weighting factors used during controller synthesis. The main area of interest is Mode 2, which has improved considerably such that $P(\zeta<5 \%)=12.3 \%$. This improvement is due to the use of the probabilistic mode location for Mode 2.

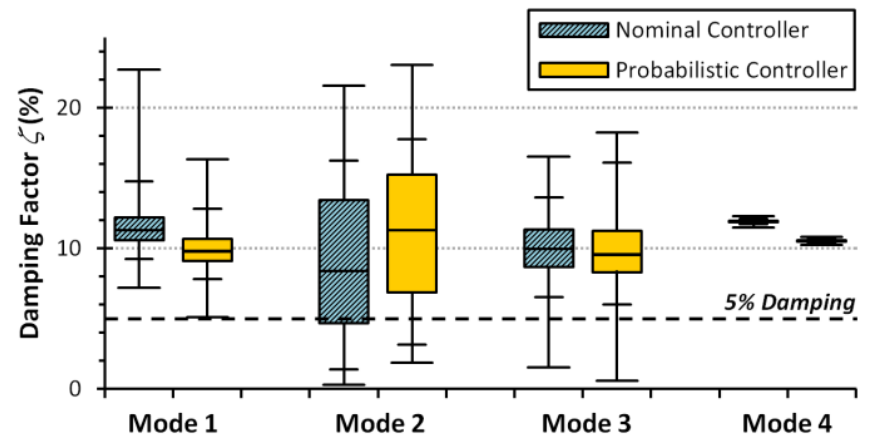

Fig. 5. Damping factors for the low frequency modes with the nominal and probabilistic controllers installed.

It should be noted that achieving $P(\zeta<5 \%)=0$ for each mode is not the direct aim of this study (although it would be desirable for optimal system performance with respect to oscillation damping). Rather, the benefits of using a probabilistic approach to controller tuning are illustrated. The simple use of the probabilistic modal location for a poorly damped Mode 2, ascertained through simulation of the known variation in operating conditions, results in a more robust damping controller.

\section{ApPlying the Probabilistic Collocation METHOD}

It can be seen that use of the probabilistic location for Mode 2 resulted in a more robust controller performance with respect to the damping of that mode. The PCM can be used in order to determine these probabilistic parameters without the need for thousands of full system linearizations (which could take a prohibitively long time to perform).

The variable network parameters identified in Section V.A constitute a set of 55 uncertainties $\Psi$ (15 generators, 35 loads, 4 VSC-MTDC nodes, and 1 wind generation unit). The number of coefficients $c$ (and therefore the required number of full system linearizations) required to produce a PCM model of order $o$ for a system with $m$ uncertain parameters is based on the binomial coefficients, and given by (8). This increases rapidly with increasing $m$, therefore it is beneficial to minimize the number of modeled uncertainties.

$$
c=\left(\begin{array}{c}
m+o \\
m
\end{array}\right)
$$

\section{A. Reduction in uncertainties using eigenvalue sensitivity}

The sensitivity of the eigenvalue of interest $\lambda_{i}$ (in this case Mode 2) to each uncertain parameter $\psi_{j} \in \Psi$ is established using the rank index $r_{\lambda_{i} \psi_{j}}$ as shown in (9). This rank is derived from one suggested in [12], though not implemented, for use when applying the PCM to large power systems.

$$
r_{\lambda_{i} \psi_{j}}=\left|\frac{\partial \lambda_{i}}{\partial \psi_{j}}\right|\left|\frac{\sigma_{\psi_{j}}}{\mu_{\psi_{j}}}\right|
$$

Where $\mu_{\psi_{j}}$ and $\sigma_{\psi_{j}}$ are respectively the mean and standard deviation of the uncertain parameter $\psi_{j}$.

Ranks were calculated for the complete set of 55 uncertain parameters for Mode 2. These rank values can be used directly to assess which parameters should be included in the reduced set of uncertainties. Quick analysis was performed to identify the parameters with an $r_{\lambda_{i} \psi_{j}}$ value greater than double the next highest rank value. Such a sharp decline in $r_{\lambda_{i} \psi_{j}}$ value between two parameters suggests a significant reduction in parameter importance. Such an interval occurs only between parameters $1-2,10-11,52-53$, and 54-55. As more than one parameter will likely be needed, and modeling 52 or more is impractical with the PCM, the results indicate that the top ten ranked parameters provide a suitable approximation.

The set of reduced parameters is as follows: $\Psi^{\text {red }}=\{$ wind, L41, L42, G15, VSC-4, VSC-3, G14, L18, G16, L50\} (presented in ranked order). The remaining parameters are modeled as constant at their nominal operating points during production of the PCM model.

\section{B. Discontinuities in wind farm power}

The power curve for a Vestas V-80 turbine [22] displays discontinuities which must be taken into consideration when using the PCM. The wind speed was the top ranked uncertainty and is certainly required as a PCM model input. The wind farm was considered as operating in three distinct regions: $(i)$ at wind speeds when no power is produced $(v<4$ $\mathrm{ms}^{-1}$, or $\left.v \geq 25 \mathrm{~ms}^{-1}\right)$, (ii) at wind speeds when power output is curtailed at the maximum wind farm capacity of $300 \mathrm{MW}$ 
$\left(18 \leq v<25 \mathrm{~ms}^{-1}\right)$, and (iii) at wind speeds when power output is variable $\left(4 \leq v<18 \mathrm{~ms}^{-1}\right)$. For each of these operational regions, a separate PCM model was produced. The correct model was then selected during the later MC runs of the PCM function based upon the randomly selected wind speed.

\section{PCM-based Probabilistic Modal Estimation}

The PCM was used to estimate the damping $\sigma$ and frequency $\omega$ of Mode 2. This was completed using the reduced uncertain parameter set $\Psi^{\text {red }}$ established previously. The PCM-based probabilistic modal location (from the produced distributions for $\sigma$ and $\omega$ ) was determined as $\lambda_{P C M}^{\text {Mode } 2}=$ $-0.122 \pm j 2.89$.

Shown in Fig. 6 are the probabilistic values for $\sigma$ and $\omega$ of Mode 2 as determined when modeling increasing numbers of uncertain parameters (according to their established rank). This has been produced to demonstrate the validity of using the top ten ranked parameters; once ten uncertain parameters are used there is little further variation in the probabilistic modal values. As only the most probable values are required for the modal tuning (the peaks of the produced distributions), accuracy can be sacrificed at the tails of the distributions and second order PCM models are sufficient. If the previously calculated $r_{\lambda_{i} \psi_{j}}$ values do not directly suggest a suitable number of uncertain parameters to include in $\Psi^{\text {red }}$ then it is possible to create models of increasing order until little variation in the probabilistic mode values is seen. This will require more computational time, on the other hand producing low order models with small numbers of parameters requires significantly fewer full linearizations and significant savings are still seen compared with the traditional MC-based approach.
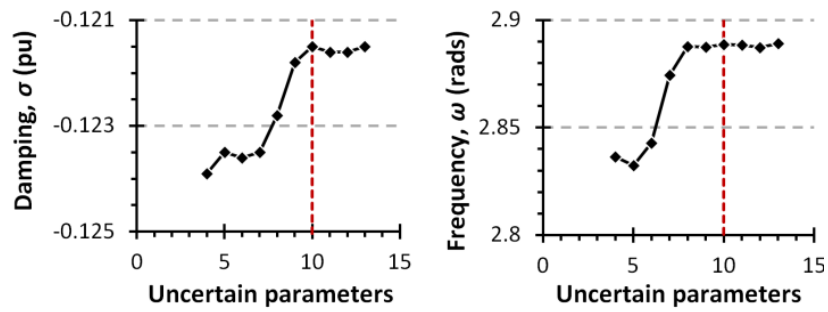

Fig. 6. PCM-based probabilistic Mode 2 values with varying numbers of modeled uncertain parameters.

By assessing the PCM-based probabilistic values for Mode 2 it can be seen that mode location is closer to that of the MCbased probabilistic location than the nominal location is. It can be seen that there is still some discrepancy between the frequency values, due to the approximations of the PCM approach.

\section{Performance of the PCM-based Probabilistic Controller}

The damping control was synthesized once more, using the PCM-based probabilistic location of Mode 2 as a demonstration of the proposed methodology. As before, the target damping was set at $20 \%$ per mode, singular value gain was limited to $6 \mathrm{~dB}$ and the final controller was reduced to $32^{\text {nd }}$ order. The PCM-based probabilistic controller was assessed against the full range of system conditions.

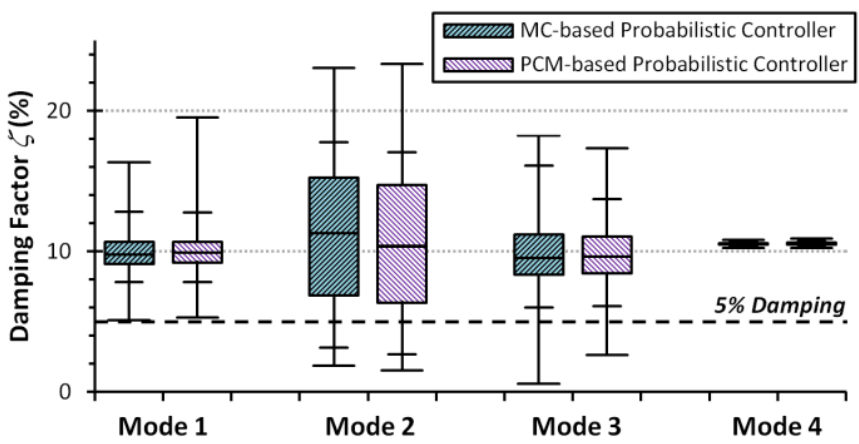

Fig. 7. Damping factors for the low frequency modes with the MC-based and PCM-based probabilistic controllers installed.

A comparison of the damping factors seen for the low frequency modes with the MC-based and PCM-based probabilistic controllers installed is presented in Fig. 7. It is clear that the two controllers perform very similarly, with only slight reduction in the damping of Mode 2. Analysis reveals that $P(\zeta<5 \%)=16.1 \%$ which is slightly increased from $12.3 \%$ seen with the MC-based probabilistic controller. However, this is still a large improvement over the standard nominal controller. The risk of poor damping for all low frequency modes with all designed controllers is summarized in Table 2.

TABLE 2

RISK OF POOR DAMPING FOR LOW FREQUENCY MODES

\begin{tabular}{lcccc}
\hline \multirow{2}{*}{ Controller } & \multicolumn{4}{c}{ Risk of Poor Damping, $\boldsymbol{P}(\zeta<5 \%)$} \\
& Mode 1 & Mode 2 & Mode 3 & Mode 4 \\
\hline \hline Nominal & $0 \%$ & $27.1 \%$ & $0.60 \%$ & $0 \%$ \\
MC-based Probabilistic & $0 \%$ & $12.3 \%$ & $1.08 \%$ & $0 \%$ \\
PCM-based Probabilistic & $0 \%$ & $16.1 \%$ & $0.96 \%$ & $0 \%$ \\
\hline
\end{tabular}

Both probabilistic controller lead to a marked reduction in the risk of poor damping for Mode 2 compared with the nominal controller. Also, they do cause a significant change in the damping of the other modes, specifically Mode 3 for which $P(\zeta<5 \%)$ remains roughly equal to $1 \%$.

The system response to a three phase fault within the NYPS region of the network cleared with the removal of an AC transmission line is illustrated in Fig. 8. It can be seen that the MC and PCM-based probabilistic controllers perform almost identically and stabilize the system much faster than when no POD modulation is used.

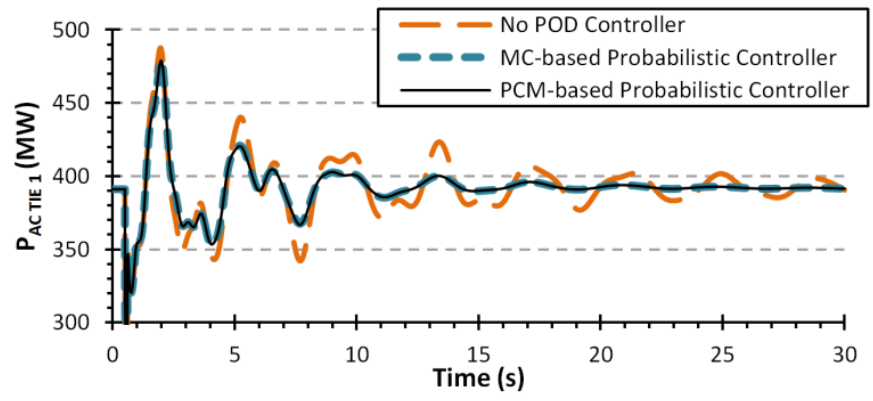

Fig. 8. Real power response of AC Tie 1 to a three phase fault within the NYPS region cleared by removing the faulted AC transmission line. 


\section{E. Computational savings using the PCM}

It has been clearly demonstrated that simply using a probabilistic representation of the system (particularly for Modes displaying poor closed loop damping) can result in improved controller robustness. However, the necessity to perform a large number of full system linearizations may prove prohibitive for large systems. The PCM method has been exploited to significantly reduce the number of full linearizations required.

Parameter ranking via eigenvalue sensitivity requires $m+1$ linearizations for a network with $m$ uncertain parameters (a change to each parameter value in turn, and the nominal case for comparison). The number of full simulations required when building each of the three PCM models required for this network (based upon the wind turbine operational region) is given by (9). The total time taken to produce the probabilistic values for Mode 2 using the PCM is detailed in Table 3. All times quoted are based upon simulations performed on a PC with $2.66 \mathrm{GHz}$ quad core CPU and $4 \mathrm{~GB}$ RAM.

TABLE 3

Process Times For THE PROBABILISTIC COLlOCATION METHOD

\begin{tabular}{lc}
\hline \multicolumn{1}{c}{ Process } & Time Taken (s) \\
\hline \hline $\begin{array}{l}\text { Eigenvalue sensitivity analysis } \\
\text { (56 full linearizations) }\end{array}$ & 190.3 \\
Identification of operating conditions & \\
for PCM model & 46.2 \\
Full system linearization & 604.2 \\
(176 full linearizations) & 1.0 \\
5000 MC runs with model function & $\mathbf{8 4 1 . 7}$ \\
\hline Total
\end{tabular}

The total time taken (841.7 s, roughly 14 minutes) is just $4.8 \%$ of the time taken to perform the full MC-based method where 5000 linearizations take roughly 17,660 s (almost 5 hours). The PCM can therefore allow quick assessment of the probabilistic modal locations with minimal loss of accuracy to enable more robust damping controller design.

Details for the different controllers are presented in Table 4. The nominal controller, designed using just one given operating condition, requires the least computational effort but clearly results in the worst system performance. The additional time and effort required in order to improve the final closed loop controller performance is clearly evident. When such controllers are required, assessment must be made as to whether the increased design effort can be afforded.

TABLE 4

DETAILS FOR VARYING CONTROLLER DESIGNS

\begin{tabular}{lcc}
\hline \multicolumn{1}{c}{ Controller } & Time Taken $(\mathbf{s})$ & Mode 2 $\boldsymbol{P}(\boldsymbol{\zeta}<\mathbf{5 \%})$ \\
\hline \hline Nominal & 3.1 & $27.1 \%$ \\
PCM-based Probabilistic & 841.7 & $16.1 \%$ \\
MC-based Probabilistic & 17,660 & $12.3 \%$ \\
\hline
\end{tabular}

It has been previously shown in [24] that the PCM is an effective tool for the rapid assessment of modal distributions. The combination of fast controller design coupled with efficient testing may facilitate the evaluation of a much greater number of controllers of varying design for a large variety of system conditions or contingency situations than would be possible using standard numerical approaches. This in turn, would lead to the selection of the most robust controllers and would ensure good practical performance.

\section{CONCLUSIONS}

This paper presented a novel probabilistic WAMS-based POD controller tuning method which can be applied to mixed AC/DC networks with a large number of uncertainties. The proposed methodology can be used to improve the robustness of a POD controller with respect to operational uncertainty when compared with a similar controller designed at the nominal operating point.

The Probabilistic Collocation Method has been used as an efficient tool to capture the probabilistic values of critical system eigenvalues which were then used to design POD controller. This resulted in over $95 \%$ computational time savings compared to a traditional Monte Carlo approach. The efficiency of the proposed technique has been demonstrated using a test network representative of future power systems a multi-area test network incorporating a six-node VSCMTDC grid with connected stochastic wind generation.

The approaches and methodologies illustrated within this paper are not limited by the controller structure, controller synthesis technique, nor the uncertainties considered. They could be implemented with any WAMS-based POD controller design which is based on a linearized power system model and with any number and type of considered uncertainties. As such, the proposed techniques are widely applicable.

Investigations using other controller designs nevertheless, would be useful in order to establish the extent to which the observed improvement in system performance is dependent on the controller structure. Potential candidates for study include $H_{\infty}$ designs which are optimized for controller robustness [15] and Model Predictive Control which can incorporate the practical operating limits of the converters [25].

The proposed probabilistic system representation established within this paper provides an efficient means of designing damping controllers which demonstrate greater robustness in uncertain power systems than if conventional deterministic approaches were used. It should be pointed out however, that this methodology cannot guarantee strict controller performance in all possible cases. Conventional, deterministic controller design procedures should be followed as required in order to guarantee a particular system response for particular operating conditions which are of concern.

\section{APPENDIX: VSC-MTDC GRID \& CONTROLLER DATA}

All data provided is based upon a 100 MW HVDC base (with $V_{D C}^{\text {base }}=500 \mathrm{kV}$ ).

$$
\begin{aligned}
& \text { VSC-MTDC Converter Capacitance (nodes 1-5) [pu]: } \\
& \quad C_{M T D C}=\{0.275,0.1875,0.1625,0.2625,0.1375,0.0750\} \\
& \text { VSC-MTDC Controller Parameters: } \\
& \quad K_{P}^{V d c}=20, K_{I}^{V d c}=200, K_{I}^{P}=50, K_{I}^{Q}=20
\end{aligned}
$$


VSC-MTDC Line Data:

\begin{tabular}{cccll}
\hline Line & From & To & R [pu] & L [pu] \\
\hline \hline 1 & 1 & 2 & 0.01 & $2.0 \times 10^{-4}$ \\
2 & 1 & 4 & 0.007 & $1.4 \times 10^{-4}$ \\
3 & 1 & 5 & 0.005 & $1.0 \times 10^{-4}$ \\
4 & 2 & 3 & 0.005 & $1.0 \times 10^{-4}$ \\
5 & 3 & 4 & 0.008 & $1.6 \times 10^{-4}$ \\
6 & 4 & 5 & 0.006 & $1.2 \times 10^{-4}$ \\
7 & 3 & 6 & 0.001 & $1.2 \times 10^{-4}$ \\
\hline
\end{tabular}

Converter stations cause active power flow losses of $1 \%$.

\section{REFERENCES}

[1] E. M. Carlini, V. G. Lopez, and M. Muller-Mienack, "Future challenges for European TSOs: a pan-European supergrid," in Cigre International Symposium on The Electric Power System of the Future, Bologna, Italy, 2011.

[2] (February 2011). Offshore Development Information Statement: Future Scenarios Consultation.

[3] D. V. Hertem, M. Ghandhari, J. B. Curis, O. Despouys, and A. Marzin, "Protection requirements for a multi-terminal meshed DC grid," in Cigre Int Symp on The Electric Power System of the Future, Bologna, Italy, 2011.

[4] J. Beerten, D. V. Hertem, and R. Belmens, "VSC MTDC Systems with a Distributed Voltage Control - A Power Flow Approach," presented at the IEEE PowerTech, , Trondheim, Norway, 2011.

[5] N. R. Chaudhuri, R. Majumder, B. Chaudhuri, and J. Pan, "Stability Analysis of VSC MTDC Grids Connected to Multimachine AC Systems," IEEE Trans on Power Delivery, vol. 26, pp. 2774-2784, 2011.

[6] R. Preece, J. V. Milanovic, A. M. Almutairi, and O. Marjanovic, "Damping of Inter-Area Oscillations in Mixed AC/DC Networks Using WAMS Based Supplementary Controller," IEEE Trans on Power Systems, vol. PP, pp. 1-10, 2012.

[7] R. Preece, J. V. Milanovic, A. M. Almutairi, and O. Marjanovic, "Probabilistic Evaluation of Damping Controller in Networks with Multiple VSC-HVDC Lines," IEEE Trans on Power Systems, vol. PP, pp. 1-10, 2012

[8] A. A. Alabduljabbar, J. V. Milanovic, and E. M. Al-Eid, "Low Discrepancy Sequences Based Optimization Algorithm for Tuning PSSs," in International Conference on Probabilistic Methods Applied to Power Systems (PMAPS), Puerto Rico, 2008.

[9] V. A. Maslennikov, J. V. Milanovic, and S. M. Ustinov, "Robust ranking of loads by using sensitivity factors and limited number of points from a hyperspace of uncertain parameters," IEEE Trans on Power Systems, vol. 17, pp. 565-570, 2002.

[10] H. Yu, C. Y. Chung, K. P. Wong, H. W. Lee, and J. H. Zhang, "Probabilistic Load Flow Evaluation With Hybrid Latin Hypercube Sampling and Cholesky Decomposition," IEEE Trans on Power Systems, vol. 24, pp. 661-667, 2009.

[11] X. Xialing, L. Tao, and Z. Xiaoming, "Probabilistic analysis of small signal stability of microgrid using point estimate method," in International Conference on Sustainable Power Generation and Supply (SUPERGEN), Nanjing, China, 2009.

[12] J. R. Hockenberry and B. C. Lesieutre, "Evaluation of Uncertainty in Dynamic Simulations of Power System Models: The Probabilistic Collocation Method," IEEE Trans on Power Systems, vol. 19, pp. 1483-1491, 2004.

[13] P. J. Davis and P. Rabinowitz, Methods of Numerical Integration. New York: Academic Press, 1975.

[14] R. D. Zimmerman, C. E. Murillo-Sanchez, and R. J. Thomas, "MATPOWER: Steady-State Operations, Planning, and Analysis Tools for Power Systems Research and Education," IEEE Trans on Power Systems, vol. 26, pp. 12-19, 2011.

[15] B. Pal and B. Chaudhuri, Robust Control in Power Systems. New York: Springer Inc., 2005.

[16] G. Rogers, Power System Oscillations. Norwell: Kluwer Academic Publishers, 2000.

[17] H. F. Latorre, M. Ghandhari, and L. Söder, "Active and reactive power control of a VSC-HVdc," Electric Power Systems Research, vol. 78, pp. 1756-1763, 2008.
[18] S. Cole, J. Beerten, and R. Belmans, "Generalized Dynamic VSC MTDC Model for Power System Stability Studies," IEEE Trans on Power Systems, vol. 25, pp. 1655-1662, 2010.

[19] A. Almutairi, "Enhancement of Power System Stability using Wide Area Measurement System Based Damping Controller," Ph.D., School of Electrical and Electronic Engineering, University of Manchester, Manchester, 2010.

S. Skogestad and I. Postlethwaite, Multivariable Feedback Control: Analysis and Design. Chichester: John Wiley \& Sons, 1996.

[21] J. P. Coelingh, A. J. M. van Wijk, and A. A. M. Holtslag, "Analysis of wind speed observations over the North Sea," Journal of Wind Engineering and Industrial Aerodynamics, vol. 61, pp. 5169, 1996.

[22] (2012). V80-2.0 MW: Unsurpassed reliability and performance at high-wind sites in North America.

[23] T. M. Mitchell, Machine Learning. New York: McGraw Hill, 1997.

[24] R. Preece, N. C. Woolley, and J. V. Milanovic, "The Probabilistic Collocation Method for Power System Damping and Voltage Collapse Studies in the Presence of Uncertainties," IEEE Trans on Power Systems, vol. PP, pp. 1-10, 2012.

[25] E. F. Camacho and C. B. Alba, Model Predictive Control, Second ed. New York: Springer, 2004.

Robin Preece ((GS'10, M'13) received his BEng degree in Electrical and Electronic Engineering in 2009 and his PhD degree in 2013, both from the University of Manchester, United Kingdom. He is currently working as a Research Associate at the same institution investigating risk and uncertainty with respect to the stability of future power systems.

Jovica V. Milanović (M'95, SM'98, F'10) received his Dipl.Ing. and his MSc degrees from the University of Belgrade, Yugoslavia, his $\mathrm{PhD}$ degree from the University of Newcastle, Australia, and his Higher Doctorate (DSc degree) from the University of Manchester, UK, all in Electrical Engineering. Currently, he is a Professor of electrical power engineering and Director of External Affairs in the School of Electrical and Electronic Engineering at the University of Manchester (formerly UMIST), UK, Visiting Professor at the University of Novi Sad, Novi Sad, Serbia and Conjoint Professor at the University of Newcastle, Newcastle, Australia. 